\title{
Changes in Doppler Waveform of Hepatic Vein in Liver Cirrhosis
}

\author{
Sharma S*, Prasad Adhikari I and Khadka H \\ Department of Radiology, Bir Hospital, National Academy of Medical Sciences, Nepal \\ *Corresponding author: Dr. Saroj Sharma, Chief consultant Radiologist, National \\ Academy of Medical Sciences, Bir Hospital, Kathmandu, Nepal, Email: \\ drsarojsharma@hotmail.com
}

\section{Research Article}

Volume 4 Issue 2

Received Date: March 13, 2019

Published Date: April 08, 2019

DOI: $10.23880 / \mathrm{ijbp}-16000152$

\section{Abstract}

Background: Cirrhosis is a common problem in Nepalese population and is a leading cause of chronic liver disease. Hepatic venous Doppler can be a tool for diagnosis of cirrhosis and to see correlation between waveform changes and severity of cirrhosis.

Objective: We aimed to see hepatic waveform and velocity changes with the severity of cirrhosis.

Methods: 69 consecutive patients of liver cirrhosis were enrolled in the study. Doppler waveforms were obtained from right hepatic vein in all the cases and classified as triphasic, biphasic or flat. Waveform comparisons were made among patients with differing grades of cirrhosis; Child Pugh score was used to assess severity of cirrhosis.

Result: Study involved 69 cirrhotic patients with age ranging from 25 to 74 years; 47(68.1\%) were male. There were 27 patients in Child-Pugh grade A, 18 patients in grade B and 24 patients in grade C. We observed that the hepatic venous waveform progressively changed from triphasic to biphasic to flat with advancing grade of cirrhosis.

Conclusion: Both maximum forward velocity and mean velocity were significantly higher in cirrhotic patients and this velocity was observed to increase with increasing severity of cirrhosis. Thus alteration in hepatic venous blood flow pattern and velocity can be a useful noninvasive tool for diagnosing patients with cirrhosis.

Keywords: Cirrhosis; Doppler waveform; Liver disease

\section{Introduction}

The Normal doppler waveforms of the hepatic veins reflect the hemodynamics of the right atrium. The waveform is triphasic; two large antegrade diastolic and systolic waves and a small retrograde wave corresponding to the atrial kick. Because the walls of the hepatic veins are thin, diseases of the hepatic parenchyma may alter their compliance. In many patients with compensated cirrhosis, the doppler waveform is abnormal. Two abnormal patterns have been described: decreased amplitude of phasic oscillations with loss of reversed flow; and a flattened waveform [1]. These abnormal patterns have also been found in patients with fatty infiltration of their liver [2].

As cirrhosis progresses, luminal narrowing of the hepatic veins may be associated with flow alterations 


\section{International Journal of Biochemistry \& Physiology}

visible on color and spectral doppler. High velocity signal through an area of narrowing produces color aliasing and turbulence [3].

Cirrhosis is characterized pathologically by diffuse parenchymal destruction, fibrosis with alteration of hepatic architecture, and innumerable regenerative nodules that replace normal liver parenchyma. Causes of cirrhosis include hepatic toxins like alcohol, drugs, infection like viral hepatitis B and C, biliary obstruction and hereditary conditions like Wilson disease. Cirrhosis has been classified as micronodular, in which nodules are 0.1 to $1 \mathrm{~cm}$. in diameter, and macronodular, characterized by nodules of varying size, up to $5 \mathrm{~cm}$ in diameter. Alcohol consumption is the most common cause of micronodular cirrhosis, and chronic viral hepatitis is the most frequent cause of macronodular form [4].

In cirrhosis, Child-Pugh grade is used to assess the hepato-cellular function. The determining five factors are: serum bilirubin, serum albumin, ascites, and encephalopathy and prothrombin time. Every patient is assigned a grade - A, B or C. Grade A is prognostically better and grade $\mathrm{C}$ is worst [5].

The Hepatic veins are relatively straight, anechoic, tubular structures that converge on the IVC approximately 1 centimeter below its confluence with the right atrium. The walls of hepatic veins are relatively hypoechoic which helps to differentiate them from the portal veins in the more echogenic portal triads. There are no valves in the hepatic veins. In most people the right, middle, and left hepatic veins enter the IVC in a crow's foot configuration when viewed in the transverse plane [6].

Cirrhosis is a common disease affecting mainly middle aged patient having significant socio-economic impact. Liver biopsy is considered gold standard for diagnosis of cirrhosis but it has several potential complications including death [5]. Thus if we can predict cirrhosis and its grade noninvasively with ultrasound then biopsy may not be necessary and complications would be avoided.

\begin{tabular}{|c|c|c|c|}
\hline Score & $\mathbf{1}$ & $\mathbf{2}$ & $\mathbf{3}$ \\
\hline Ascites & None & Mild & Moderate/Severe \\
\hline Encephalopathy & None & Mild & Marked \\
\hline Bilirubin (mg/dl) & $<2$ & $2-3$ & $>3$ \\
\hline Albumin (g/dl) & $>3.5$ & $2.8-3.5$ & $<2.8$ \\
\hline $\begin{array}{c}\text { Prothrombin time } \\
\text { (seconds over normal) }\end{array}$ & $<4$ & $4-6$ & $>6$ \\
\hline
\end{tabular}

Table 1: Child-Pugh Score.

\begin{tabular}{|c|c|c|c|}
\hline & \multicolumn{3}{|c|}{ \% Survival } \\
\hline Grade(Score) & 1 year & 5 years & 10 years \\
\hline Child's A (< 7) & 82 & 45 & 25 \\
\hline Child's B ( 7- 9) & 62 & 20 & 7 \\
\hline Child's C ( 10+) & 42 & 20 & 0 \\
\hline
\end{tabular}

Table 2: Above scores are added for survival figures below.

\section{Methodology}

The study was done in National academy of medical sciences, Bir hospital for period of 1 year. 72 patients above the age of 15 years with clinical, laboratory and radiological evidence of cirrhosis were included in the study (histopathological confirmation not available as it is commonly not done). Out of 72 cases 3 cases having liver SOLs during Doppler were excluded from the study. Patients with cardiac diseases including cor pulmonale were excluded from study. The diagnosis of cirrhosis was based on clinical and biochemistry parameters and imaging findings. The severity of liver cirrhosis was assessed by Child-Pugh scoring system.

Patient for study was placed in supine position in bed. Doppler study was done in right hepatic vein after clear visualization of all hepatic veins. Examination was done in quiet normal respiration with short period of breath holding whenever needed. Examination was done with 3.6 and $6.2 \mathrm{MHz}$ curvilinear and linear probes of Seimens model Acuson X150 and Sonosite Micromax ultrasound machines.

Pulse repetition frequency was adjusted so as not to surpass the limit of the displayed maximum velocity. The angle of insonation was always maintained $<60^{\circ}$ and Doppler gate was placed in mid of the vessel.

Right hepatic vein and its confluence with inferior venacava were identified. Waveforms of $\mathrm{HV}$ were obtained by positioning the sample volume within $2-3 \mathrm{~cm}$ from the opening into the inferior venacava. Spectral Doppler waveforms were obtained and Doppler waveforms were divided into three types. The waveforms were considered triphasic when there were two hepatofugal or antegrade phases (related to atrial and ventricular diastole) and a short phase of hepatopetal or retrograde flow (caused by the pressure increase in the right atrium at atrial systole). Biphasic waveforms lacked negative wave and showed oscillation of positive waves. Flat waveforms were those without any phasic oscillation. Three readings were taken for velocity measurements and mean of three was noted as final reading. 


\section{International Journal of Biochemistry \& Physiology}

\section{Results}

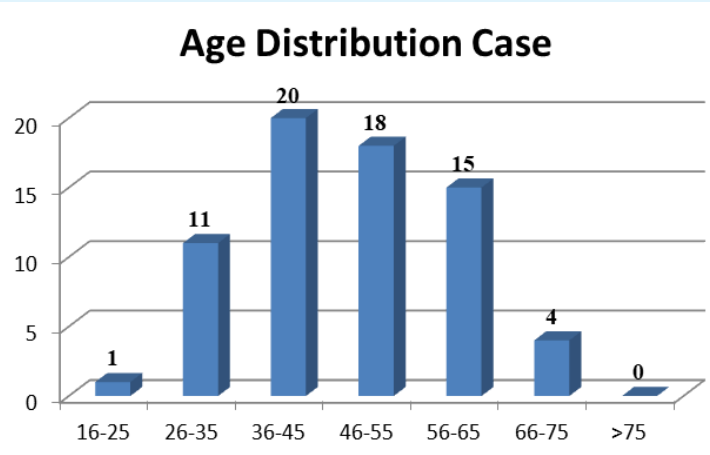

Figure 1: Age Distribution of Cases (cirrhotics).

There were total of 69 cases. The youngest and oldest patients were of age 25 and 74 years respectively. Most of the patients were in the age group 36-45 years (28.9\%). Only patients of more than 15 years were included in the study.

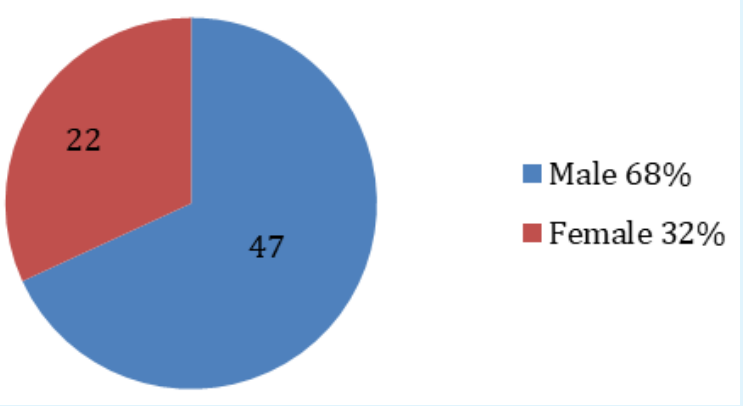

Figure 2: Sex distribution.

Cirrhosis was more common in male patients. Out of 69 patients 47 were male and 22 were female with, a male: female ratio 2.1:1.



Figure 3: Distribution of etiological factors.
Detail history was taken for various etiological factors of cirrhosis. Alcohol was the most common etiological factor and accounts for 56 cases of total 69 . Viral hepatitis was causative factor in 7 cases. In 5 cases causative factor was unknown.

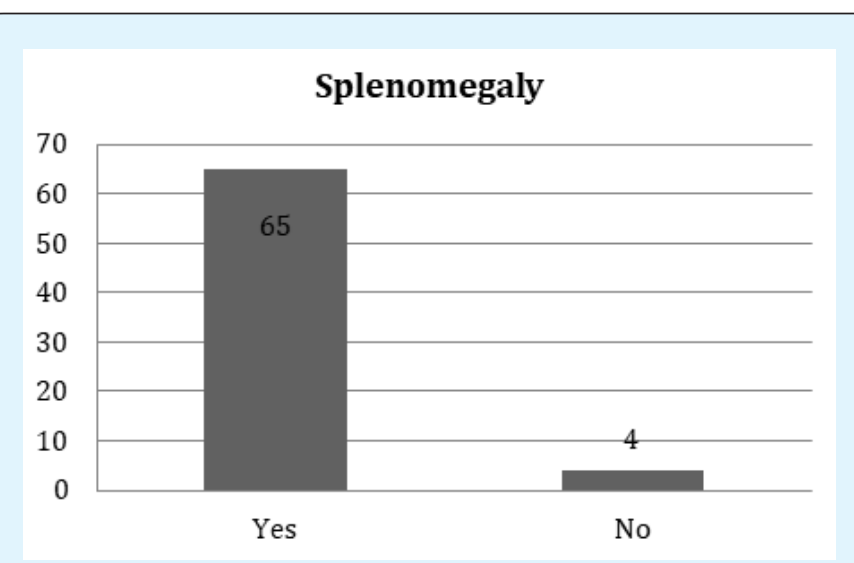

Figure 4: Distribution of splenomegaly among cirrhotic cases.

Spleen enlargement was common in cirrotic patients secondary to portal hypertension. Out of 69 cirrhotic cases Splenomegaly was present in $94.2 \%$. In 4 cases spleen was normal in size. Splenic size more than $12 \mathrm{~cm}$ longitudinally was graded as splenomegaly.

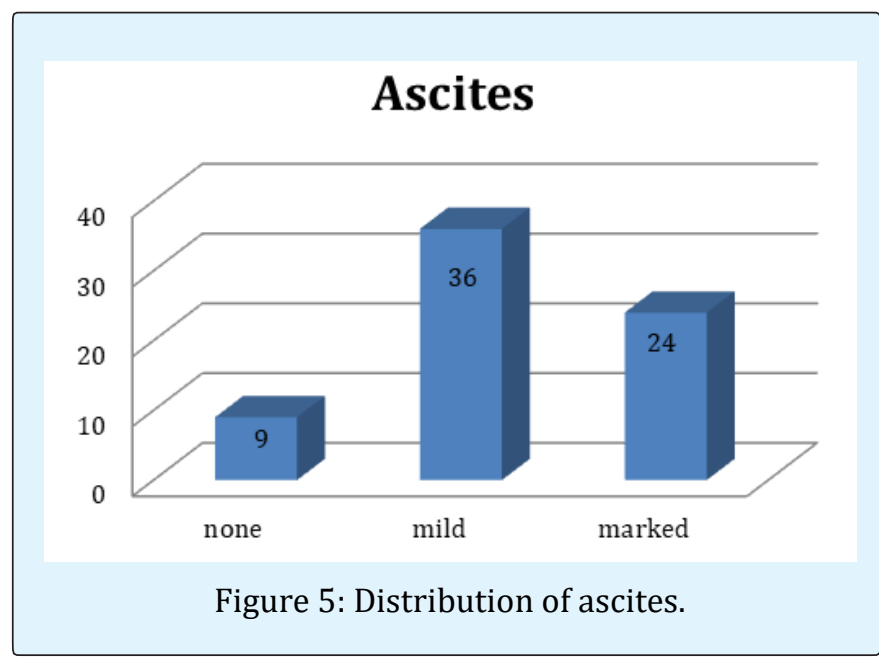

Ascites had common association with cirrhotic patients. It was common findings in cirrhosis. In our study 36 cases had mild ascites (described as fluid collection only in pelvic cavity and 24 cases had marked ascites. Out of 69 cases ascites was absent in 9 cases. So Out of 69 patients $60(86.9 \%)$ patients had ascites. 


\section{International Journal of Biochemistry \& Physiology}

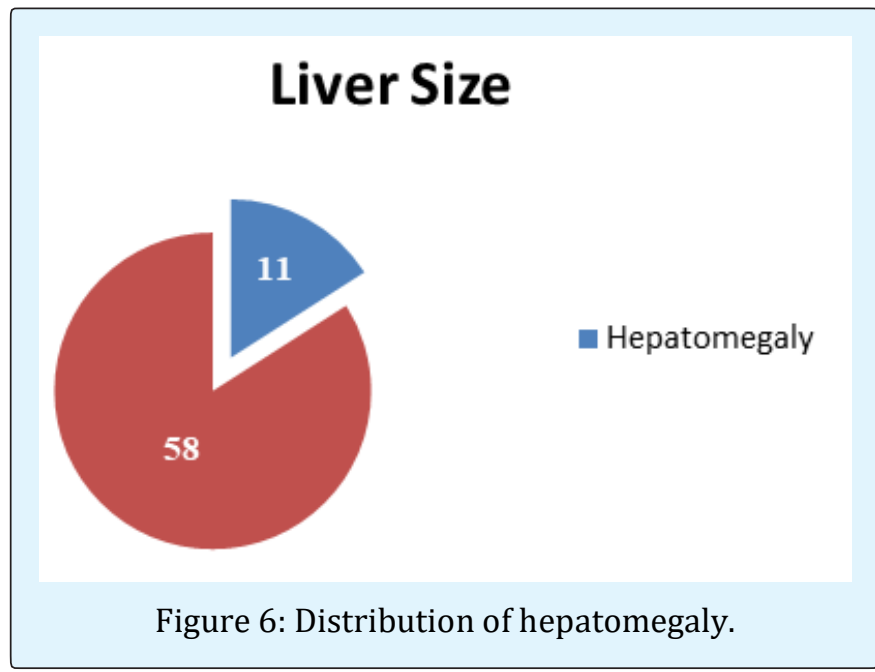

Size of liver more than $15 \mathrm{~cm}$ in craniocaudal direction is categorized as hepatomegaly. Hepatomegaly is common in early stage of cirrhosis and in hepatitis stage of liver parenchymal alteration. Out of 69 patients in our study only $11(15.9 \%)$ patients had hepatomegaly.

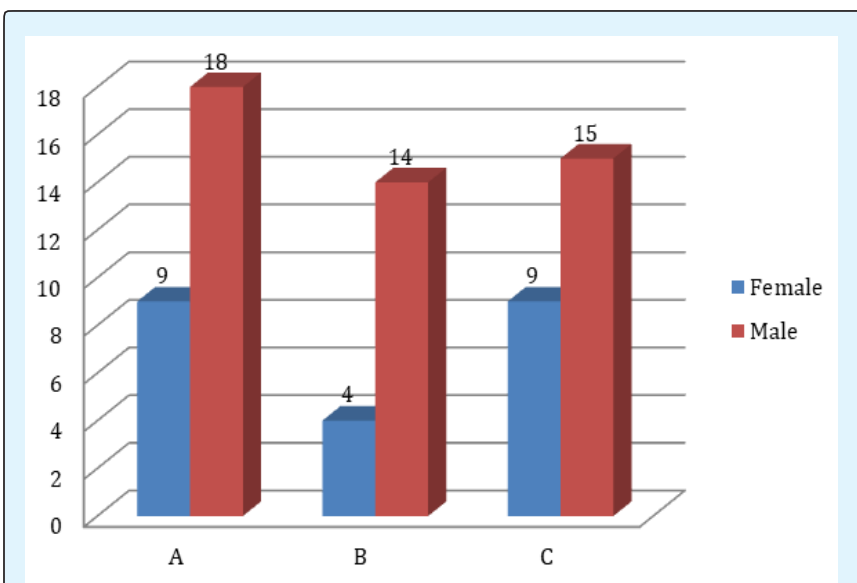

Figure 7: Distribution of patients by Child-Pugh grade.

Out of 69 cases 27 were of Child-Pugh grade A, 18 in B and 24 in C. That is 27 patients had child - Pugh score less than 7. They have more years of survival than those in Grade B and C. 18 patients were under Grade B which child Pugh score is 7-9. 24 patients in Grade C with child Pugh score 10 or more had more severity of disease and prognosis is poor.

\begin{tabular}{|c|c|c|c|c|}
\hline \multirow{2}{*}{ Pattern of waveform } & \multicolumn{4}{|c|}{ Cirrhotic group (N=69) } \\
\cline { 2 - 5 } & Total & Child's A (n= 27) & Child's B (n=18) & Child's C (n=24) \\
\hline Triphasic & $30(43.47 \%)$ & $15(55.55 \%)$ & $9(50 \%)$ & $6(25 \%)$ \\
\hline Biphasic & $32(46.37 \%)$ & $10(37 \%)$ & $7(38.8 \%)$ & $15(62.5 \%)$ \\
\hline Flat & $7(10.14 \%)$ & $2(7.4 \%)$ & $2(11.11 \%)$ & $3(12.5 \%)$ \\
\hline
\end{tabular}

Table 2: Pattern of hepatic vein waveforms in Cirrhotics with Child's grading

In the cirrhotic patients biphasic waveform was more common than the triphasic waveform. Also, proportion of patients with biphasic waveform increased with increasing severity of cirrhosis.

\begin{tabular}{|c|c|c|c|c|c|c|}
\hline Parameter & $\begin{array}{c}\text { Grade A } \\
\text { Mean } \pm \text { SD }\end{array}$ & $\begin{array}{c}\text { Grade B } \\
\text { Mean } \pm \text { SD }\end{array}$ & $\begin{array}{c}\text { Grade C } \\
\text { Mean } \pm \text { SD }\end{array}$ & Degree of freedom & F & P value \\
\hline Maximum forward velocity (cm/s) & $27.6 \pm 5.1$ & $31.3 \pm 5.6$ & $36.0 \pm 6.2$ & 22,1 & 1.6 & 0.550 \\
\hline Mean velocity (cm/s) & $8.0 \pm 2.3$ & $9.4 \pm 2.6$ & $13.4 \pm 3.5$ & 19.4 & 0.9 & 0.600 \\
\hline
\end{tabular}

Table 3: Right hepatic venous blood velocity in different grades of severity of cirrhosis (Child-Pugh Score) ANOVA test

As the severity of cirrhosis increased both maximum forward velocity and mean velocity increased. However the change was not statistically significant.

\section{Discussion}

The normal Doppler waveforms of the hepatic veins reflect the hemodynamics of the right atrium. The normal waveform is triphasic: two large antegrade diastolic and systolic waves and a small retrograde wave corresponding to the the atrial kick. Because the walls of the hepatic veins are thin, diseases of the hepatic parenchyma may alter their compliance. In many patients with compensated cirrhosis, the doppler waveform is abnormal. Two abnormal patterns have been described: decreased amplitude of phasic oscillations with loss of reversed flow; and a flattened waveform [1]. The phasicity is caused by changes in cardiac cycle, predominantly the change in right atrial pressure [7]. 


\section{International Journal of Biochemistry \& Physiology}

In our study, doppler study of right hepatic vein was performed in cirrhotic patients admitted in medical ward of our hospital during the study period of one year. B mode, color Doppler and spectral doppler were used for the examination. A total number of 69 cirrhotic patients were enrolled on the study.

Patients of more than 15 years were included in the study. The youngest and oldest patients in our study were 25 years and 74 years respectively. Within this age range, highest number of patients fell in the age range of 36 to 45 years. Mean age of the case was 48 years and that of control was 41 yrs. Male patients contributed majority of study group, $68.11 \%(47)$ with female patient in minority, $31.89 \%$ (22). Male to female ratio was 2.1:1. The reason behind male predominance in cirrhosis could be more prevalence of male alcoholics than female.

Alcohol was the most common cause of cirrhosis; it was implicated in 56 patients, i.e. $81.1 \%$ of total cases. Other causes were hepatitis B virus 4 patients, hepatitis C virus 2 patients, combined alcohol and hepatitis $C$ viral hepatitis in 1 patient, cryptogenic or idiopathic in 1 patient and in 5 cases no cause could be determined.

Splenomegaly was present in $94.2 \%$ (64) cases while hepatomegaly was present in $15.9 \%$ (11) patients. For the presence of ascites patients were divided into none, mild and marked. 9 patients did not have ascites, 36 cases had mild ascites and 24 cases had marked ascites.

In our study, out of 69 patients, $43.47 \%$ (30) patients showed triphasic waveform; $46.37 \%$ (32) patients showed biphasic waveform and $10.14 \%$ (7) cases showed flat waveform. Change of waveform from triphasic to biphasic seems indicative of some changes in the liver parenchyma as cirrhotic patients showed change from triphasic to biphasic or flat waveform. Although the phasicity is cardiac in origin, predominantly the change in right atrial pressure changes, respiratory motion can alter the HV waveforms and its components [8]. Bolondi, et al. [1] have reported that the underlying mechanism of the change in the hepatic vein waveform may be related to liver fibrosis, which progressively reduces phasic oscillation in hepatic veins. In another study by Kawanka $\mathrm{H}$, et al. in Japan, they found that normal or triphasic waveform in 34/103 patients and they have also concluded that the liver parenchyma is not the only factor that causes changes in waveform [9].

In the study done by Bolondi L, et al. [1] all controls showed triphasic waveforms. $50 \%$ of patients showed triphasic waveforms, however $31.7 \%$ patients showed biphasic waveform and in their study monophasic waveform was more common than in our study which was $18.3 \%$. Colli, et al. [2] found that $61.5 \%$ of patients showed abnormal waveforms in hepatic venous Doppler study, their findings were also comparable to our study. Our study had similar findings as in study done by KC S et al. [10] In their study out of 120 cirrhotic patients, 50\% showed triphasic waveforms, $46.6 \%$ showed biphasic waveforms and only $3.4 \%$ showed flat waveforms.

In another study done by Von Herbay, et al. [11] 40\% of patients showed triphasic waveforms. However only $16 \%$ of patients had biphasic waveforms. But in their study flat waveform was more common than in our study. They found flat waveform in $43 \%$ of patients. With Valsalva maneuver and suspended respiration, the negative oscillation disappeared in few cases and it may be the reason of discrepancy in findings among many studies. This may be the reason why $7 / 75$ (9.33\%) subjects of Shapiro, et al. [12] showed flat waveform even without liver or cardiac disease. Changes in the right atrial pressure with respiratory maneuvers may be a probable cause. However, it was seen in only certain cases and the reason is not well understood why it occurs in some patients only. In another study by Pedersen, et al. [13] abnormal waveform was found in 17/139 studied patients who were not having any liver disease.

In our study there were 27 patients in Child-Pugh grade $A, 18$ patients in grade $B$ and 24 patients in grade $C$. Of the 27 patients in Child-Pugh grade A, 55.5\% (15) cases showed triphasic waveforms, 37.0\% (10) patients showed biphasic waveforms and $7.4 \%$ (2) patients showed flat waveforms. Of the 18 patients in Child-Pugh grade B 50\% (9) patients showed triphasic waveforms, $38.8 \%$ (7) patients showed biphasic waveforms and $11.1 \%$ (2) patients showed flat waveforms. Out of 24 patients in Child-Pugh grade C 25\% (6) patients showed triphasic waveform, $62.5 \%$ (15) patients showed biphasic waveforms and $12.5 \%$ (3) patients showed flat waveforms. We observed that the hepatic venous waveform progressively changed from triphasic to biphasic to flat with advancing grade of cirrhosis. This could be because of the increasing severity of architectural distortion of liver parenchyma with increasing grade of cirrhosis. However statistical analysis showed this to be insignificant.

KC S, et al. [10] found that out of 54 patients in ChildPugh grade A, 59.3\% patient showed triphasic waveform, $37 \%$ had biphasic waveform and $3.7 \%$ had flat waveform. Out of 35 patients in Child-Pugh grade B, they found that $54.2 \%$ patient showed triphasic waveform, $42.9 \%$ patient showed biphasic waveform however only $2.9 \%$ patients 


\section{International Journal of Biochemistry \& Physiology}

showed flat waveform. Similarly out of 31 patients in Child-Pugh grade C, 29\% patients showed triphasic waveform, $67.8 \%$ patient had biphasic waveform and $3.2 \%$ showed flat waveform. This finding was comparable to our study except that flat waveform is slightly more common in our study. Blanco et al also found no statistically significant difference between the different Doppler waveform pattern and Child-Pugh score.

In line with other studies, maximum forward velocity was significantly elevated in liver cirrhotic patients. As cirrhosis progresses, luminal narrowing of the hepatic veins may be associated with flow alterations visible on color and spectral doppler. High velocity signal through an area of narrowing produces color aliasing and turbulence [3].

On comparing maximum forward velocity among different grades of cirrhosis we found that maximum forward velocity in Child-Pugh grade A was $27.6 \pm 5.1 \mathrm{~cm} / \mathrm{s}$, in $B$ it was $31.3 \pm 5.6 \mathrm{~cm} / \mathrm{s}$ and in $C$ it was $36.0 \pm 6.2 \mathrm{~cm} / \mathrm{s}$ with P-value 0.550 . It was observed that as the severity of cirrhosis increased, the maximum forward velocity was also increased. In the similar study done by $\mathrm{KC} \mathrm{S}$, et al. [10] maximum forward velocity in Child-Pugh grade A was $31.8 \pm 11.4 \mathrm{~cm} / \mathrm{s}$, in Child-Pugh grade B it was $40.2 \pm 16.4 \mathrm{~cm} / \mathrm{s}$ and in Child-Pugh grade $\mathrm{C}$ it was $48.8 \pm 17.5 \mathrm{~cm} / \mathrm{s}$ which also supports our study.

On comparing mean velocity of hepatic vein in different grades of cirrhosis we found that it was $8.0 \pm 2.3 \mathrm{~cm} / \mathrm{s}$ in child pugh grade $A, 9.4 \pm 2.6 \mathrm{~cm} / \mathrm{s}$ in grade $B$ and $13.4 \pm 3.5 \mathrm{~cm} / \mathrm{s}$ in grade $C$ patients with $\mathrm{p}$-value 0.60 . It was observed that as severity of cirrhosis increased mean hepatic blood velocity also increased. In the similar study by KC S, et al. [10] they found that mean hepatic vein velocity Child-Pugh grade A was $9.7 \pm 4.1$, in grade $B$ it was $11.6 \pm 5.4 \mathrm{~cm} / \mathrm{s}$ and in grade $C$ it was $19.1 \pm$ $10.3 \mathrm{~cm} / \mathrm{s}$. They also observed that poorer the grade of cirrhosis, greater was the mean hepatic venous velocity.

Continuous flow of RHV due to increased flow into the right lobe can be the plausible explanation of disappearance of negative waves and decrease in phasic oscillations of $\mathrm{HV}$ in the cirrhotic patients. This can be explained by hyperdynamic circulation present in liver cirrhosis [14,15] and arterialization of the cirrhotic liver[16].

Among cirrhotics, non-triphasic group had increased hepatic inflow and all the indices of inflow were significantly higher. In normal subjects, there is no direct communication between periphery of the portal and HVs.
Blood flows from the portal venules into the HV system traversing through sinusoids. However, in pathological condition like liver cirrhosis, a direct communication occurs between the two venous systems. ${ }^{14}$ Moreover, it has been hypothesized that functionally inactive preexisting arterio-venous shunts are opened in a response to intense vasodilatation [14]. These shunts are not easily detected by conventional B mode ultrasound. They assumed that the alteration in hemodynamics of portal vein and hepatic artery had an influence on HV, its flow and waveform when there is development of abnormal communications. There is also deranged autoregulation of sinusoidal flow in cirrhotic liver.

Thus we can see that alterations in hepatic venous waveform and flow velocities are different in different grades of cirrhotic patients. This may be employed to the benefit of patients in that hepatic venous Doppler study may be used as a non-invasive tool to support the diagnosis of grade of cirrhosis.

\section{Conclusion}

There are three patterns (tripasic, biphasic and flat) of waveforms in hepatic venous Doppler study. Biphasic waveforms were more common followed by triphasic waveforms in cirrhotic group patients. As the severity of cirrhosis increases alteration of wavepattern occurs. Percentage of flat waveforms rises as liver cirrhosis severity changes from Child's Pugh grade A to grade B and to grade $\mathrm{C}$. Both maximum forward velocity and mean velocity were higher with increasing severity of cirrhosis.

So we would like to infer that study of alteration in hepatic venous waveform pattern and increase in velocity can be a useful noninvasive tool to distinguish patients in different grades of cirrhosis.

However, because of the small sample size definite conclusion cannot be drawn. We would like to recommend further well-designed, multi-institutional trials with larger number of patients to further evaluate the feasibility of the study.

Acknowledgements: We would like to thank the Dr. Niranjan Koirala, Dr. Koirala Research Institute for Biotechnology and Biodiversity, Kathmandy, Nepal for his valuable technical, managerial and publication support.

Conflict of Interests: All the authors declare that there is no conflict of interest. 


\section{International Journal of Biochemistry \& Physiology}

Funding: The research case was supported by Bir Hospital, NAMS, Kathmandu, Nepal.

\section{References}

1. Bolondi L, Li Bassi S, Gaiani S, Zironi G, Benzi G, et al. (1991) Liver cirrhosis: Changes of Doppler waveform of hepatic veins. Radiology 178(2): 513-516.

2. Colli A, Cocciolo M, Riva C, Martinez E, Prisco A, et al. (1994) Abnormalities of Doppler waveform of the hepatic veins in patients with chronic liver disease: correlation with histologic findings. AJR 162(4): 833837.

3. Wilson SR, Withers CE (2005) The liver. In: Rumack CM, Wison SR, Charbonean JW (Eds.), Diagnostic Ultrasound. $3^{\text {rd }}$ (Edn.), China: Elsevier Mosby, pp: 101.

4. Brant WE, Helms CA (1999) Fundamentals of Diagnostic Radiology. $2^{\text {nd }}$ (Edn.), Williams and Wikins, USA, pp: 671-672.

5. Paul A, Paul D, Myron P, Norman W (2006) Clinical Doppler ultrasound. $2^{\text {nd }}$ (Edn.), UK: Churchill Livingstone Elsevier, pp: 148-149.

6. Abu-Yousef MM (1992) Normal and respiratory variation of the hepatic and portal venous duplex Doppler waveforms with simultaneous electrocardiographic correlation. J Ultrasound Med 11(6): 363-268.

7. Baik SK, Kim JW, Kim HS, Kwon SO, Kim YJ, et al. (2006) Recent variceal bleeding: Doppler US hepatic vein waveform in assessment of severity of portal hypertension and vasoactive drug response. Radiology 240(2): 574-580.

8. Kawanaka H, Kinjo N, Anegawa G, Yoshida D, Migoh S, et al. (2008) Abnormality of the hepatic vein waveforms in cirrhotic patients with portal hypertension and its prognostic implications. J Gastroenterol Hepatol 23(7PT2): e129-36.

9. KC S, Matsutani S, Maruyama H, Akiike T, Saisho H (2006) Doppler study of hepatic vein in cirrhotic patients: Correlation with liver dysfunction and hepatic hemodynamics. World J Gastroenterol 12(36): 5853-5858.

10. Herbay AV, Frieling T, Haussinger D (2001) Association between duplex Doppler sonographic flow pattern in right hepatic vein and various liver diseases. J Clin Ultrasound 29(1): 25-30.

11. Shapiro RS, Winsberg F, Maldjian C, Stancto-Pasik A (1993) Variability of hepatic vein Doppler tracings in normal subjects. J Ultrasound Med 12(12): 701-703.

12. Pedersen JF, Dakhil AZ, Jensen DB, Søndergaard B, Bytzer P (2005) Abnormal hepatic vein Doppler waveform in patients without liver disease. $\mathrm{Br} \mathrm{J}$ Radiol 78(927): 242-244.

13. Lomus DJ (2008) The Liver. In: Adam A, Dixon AK. Diagnostic Radiology A Textbook of Medical Imaging. $5^{\text {th }}($ Edn.), China: Elsevier Churchill Livingstone, pp: 725.

14. Ryan S, McNicholas M, Eustace S (2004) Anatomy for Diagnostic Imaging. $2^{\text {nd }}(E d n)$, London: Saunders, pp: 173-174.

15. Kim MY, Baik SK, Park DH, Lim DW, Kim JW, et al. (2007) Damping index of Doppler hepatic vein waveform to assess the severity of portal hypertension and response to propranolol in liver cirrhosis: a prospective nonrandomized study. Liver Int 27(8): 1103-1110. 